\title{
The Ultraluminous X-Ray Sources Population of the Galaxy NGC 7456
}

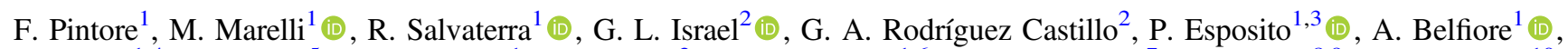

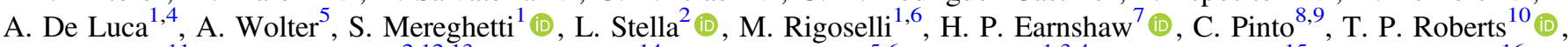

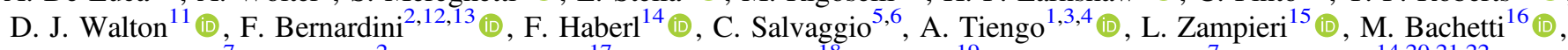
M. Brightman ${ }^{7}$, P. Casella ${ }^{2}$, D. D’Agostino ${ }^{17}$ (D) S. Dall'Osso ${ }^{18}$, F. Fürst ${ }^{19}$ (1D), F. A. Harrison ${ }^{7}$, M. Mapelli ${ }^{14,20,21,22}$ (1), A. Papitto ${ }^{2}$ (1) and M. Middleton ${ }^{23}$ (1)

${ }^{1}$ INAF-IASF Milano, via A. Corti 12, I-20133 Milano, Italy; fabio.pintore@inaf.it

2 INAF-Osservatorio Astronomico di Roma, via Frascati 33, I-00078 Monteporzio Catone, Italy

${ }^{3}$ Scuola Universitaria Superiore IUSS Pavia, piazza della Vittoria 15, I-27100 Pavia, Italy

${ }^{4}$ Istituto Nazionale di Fisica Nucleare (INFN), Sezione di Pavia, via A. Bassi 6, I-27100 Pavia, Italy

${ }^{5}$ Osservatorio Astronomico di Brera, INAF, via Brera 28, I-20121 Milano, Italy

${ }^{6}$ Dipartimento di Fisica G. Occhialini, Università degli Studi di Milano Bicocca, Piazza della Scienza 3, I-20126 Milano, Italy

${ }^{7}$ Cahill Center for Astronomy and Astrophysics, California Institute of Technology, 1216 East California Boulevard, Pasadena, CA 91125, USA

${ }^{8}$ European Space Research and Technology Centre (ESTEC), ESA, Keplerlaan 1, 2201 AZ Noordwijk, The Netherlands 9 INAF-IASF Palermo, Via U. La Malfa 153, I-90146 Palermo, Italy

${ }^{10}$ Centre for Extragalactic Astronomy, Department of Physics, Durham University, South Road, Durham DH1 3LE, UK

${ }^{11}$ Institute of Astronomy, Science Operations Department, University of Cambridge, Madingley Road, Cambridge CB3 0HA, UK

${ }^{12}$ INAF-Osservatorio Astronomico di Capodimonte, salita Moiariello 16, I-80131 Napoli, Italy

${ }^{13}$ New York University Abu Dhabi, Saadiyat Island, PO Box 129188 Abu Dhabi, United Arab Emirates

${ }_{15}^{14}$ Max-Planck-Institut für extraterrestrische Physik, Gießenbachstraße 1, D-85748 Garching, Germany

15 INAF-Osservatorio Astronomico di Padova, vicolo dell'Osservatorio 5, I-35122 Padova, Italy

${ }^{16}$ INAF-Osservatorio Astronomico di Cagliari, via della Scienza 5, I-09047 Selargius (CA), Italy

${ }^{17}$ Istituto di Matematica Applicata e Tecnologie Informatiche (IMATI) 'E. Magenes', CNR, via de Marini 6, I-16149 Genova, Italy

${ }^{18}$ Department of Physics and Astronomy, Stony Brook University, Stony Brook 11579 NY, USA

${ }^{19}$ European Space Astronomy Centre (ESAC), ESA, Camino Bajo del Castillo s/n, Villanueva de la Cañada, E-28692 Madrid, Spain

20 Dipartimento di Fisica e Astronomia "Galileo Galilei," Università di Padova, via F. Marzolo 8, I-35131 Padova, Italy

${ }^{21}$ Institut für Astro- und Teilchenphysik, Universität Innsbruck, Technikerstrasse 25/8, A-6020, Innsbruck, Austria

${ }^{22}$ Istituto Nazionale di Fisica Nucleare (INFN), Sezione di Padova, via F. Marzolo 8, I-35131 Padova, Italy

${ }^{23}$ Department of Physics and Astronomy, University of Southampton, Highfield, Southampton SO17 1BJ, UK

Received 2019 December 12; revised 2020 January 14; accepted 2020 January 20; published 2020 February 26

\begin{abstract}
Ultraluminous X-ray sources (ULXs) are a class of accreting compact objects with X-ray luminosities above $10^{39} \mathrm{erg} \mathrm{s}^{-1}$. The ULX population counts several hundred objects but only a fraction are well studied. Here we present a detailed analysis of all ULXs hosted in the galaxy NGC 7456. It was observed in X-rays only once in the past (in 2005) by XMM-Newton. but the observation was short and strongly affected by high background. In 2018, we obtained a new, deeper ( $~ 90 \mathrm{ks})$ XMM-Newton observation that allowed us to perform a detailed characterization of the ULXs hosted in the galaxy. ULX-1 and ULX-2, the two brightest objects $\left(L_{X} \sim 6-10 \times 10^{39} \mathrm{erg} \mathrm{s}^{-1}\right.$ ), have spectra that can be described by a model with two thermal components, as often found in ULXs. ULX-1 also shows one order of magnitude in flux variability on short-term timescales (hundreds to thousands of kiloseconds). The other sources (ULX-3 and ULX-4) show flux changes of at least an order of magnitude, and these objects may be candidate transient ULXs, although longer X-ray monitoring or further studies are required to ascribe them to the ULX population. In addition, we found a previously undetected source that might be a new candidate ULX (labeled as ULX-5), with a luminosity of $\sim 10^{39} \mathrm{erg} \mathrm{s}^{-1}$ and hard power-law spectral shape, whose nature is still unclear and for which a background active galactic nucleus cannot be excluded. We discuss the properties of all the ULXs in NGC 7456 within the framework of super-Eddington accretion onto stellar-mass compact objects. Although no pulsations were detected, we cannot exclude that the sources host neutron stars.
\end{abstract}

Unified Astronomy Thesaurus concepts: X-ray astronomy (1810); X-ray binary stars (1811); X-ray sources (1822); Neutron stars (1108); Pulsars (1306)

\section{Introduction}

Ultraluminous X-ray sources (ULXs) are a class of accreting compact objects in binary systems, characterized by assumed isotropic luminosities $L_{X}>10^{39} \mathrm{erg} \mathrm{s}^{-1}$ (e.g., Fabbiano 1989; Feng \& Soria 2011; Kaaret et al. 2017), i.e., about the Eddington limit for spherical hydrogen accretion onto a $10 M_{\odot}$ black hole (BH). The ULX population consists of several hundreds of sources in nearby galaxies (e.g., Earnshaw et al. 2019), and they are usually extragalactic (although see WilsonHodge et al. 2018), off-nuclear, and point-like objects.
Nowadays, they are believed to be mostly stellar-mass BHs or neutron stars (NSs) accreting well above the Eddington limit rather than sub-Eddington accreting intermediate-mass BHs $\left(10^{2}-10^{5} M_{\odot}\right.$; e.g., Colbert \& Mushotzky 1999; Farrell et al. 2009). ULXs strongly challenge our understanding of the accretion processes, especially after the discovery of at least six pulsating ULXs (PULXs) in M82 X-2 (Bachetti et al. 2013), NGC 5907 ULX-1 (Israel et al. 2017), NGC 7793 P13 (Fürst et al. 2016; Israel et al. 2017), NGC 300 ULX-1 (Carpano et al. 2018), M51 ULX-7 (Rodríguez Castillo et al. 2019), and NGC 
1313 X-2 (Sathyaprakash et al. 2019), plus the NS candidate in M51 ULX-8 showing a transient cyclotron line (Brightman et al. 2018). It is now a matter of debate whether the ULX populations preferentially host NSs more frequently than BHs (e.g., Middleton \& King 2017; Wiktorowicz et al. 2017). It has been proposed that the number of ULXs hosting NSs can be significantly higher than the observed one (King 2009; Koliopanos et al. 2017; Pintore et al. 2017; Walton et al. 2018). On the other hand, the detection of $\sim 30 M_{\odot}$ BHs by LIGO and Virgo (e.g. Abbott et al. 2016, 2019) has revived the possibility that some ULXs are powered by $>20 M_{\odot}$ BHs (e.g., Mapelli et al. 2009; Zampieri \& Roberts 2009; Mapelli et al. 2010, 2013).

In the case of super-Eddington accretion, it is expected that the accretion disk can eject radiatively powerful outflows/ winds (e.g., Poutanen et al. 2007), which, according to magneto-hydrodynamical simulations, should be turbulent (e.g., Takeuchi et al. 2013). The photospheres of these winds may be associated with the soft, thermal component (temperatures of $\sim 0.1-0.5 \mathrm{keV})$ usually observed at low energies in the ULX spectra (Gladstone et al. 2009). In addition, the spectra are often accompanied by a hard, thermal-like component with a rollover at 3-7 keV (e.g., Gladstone et al. 2009), often interpreted either as an optically thick corona around the compact object or as the inner regions of a geometrically thick accretion disk (e.g., Kawaguchi 2003). When NuSTAR spectra with high signal-to-noise ratios above $10 \mathrm{keV}$ are available, the vast majority of ULXs are also characterized by a third hard component, phenomenologically described with a cutoff power-law model, dominant in the case of the PULXs, and for this reason, possibly associated with the emission of the accretion column above the NS magnetic polar caps (e.g., Walton et al. 2018).

The short-term temporal variability properties of the ULXs differ significantly from source to source, but also from observation to observation of the same source. It has been shown that the most variable sources are generally those with the softest spectra (e.g., Sutton et al. 2013). Earnshaw et al. (2019) examined the 3XMM-DR4 catalog, which contains XMM-Newton observations performed before 2012, finding that the number of ULXs with high intra-observation variability is quite limited (eight sources) if compared to the whole population number. In addition, Heil et al. (2009) showed that the absence of high short-term variability cannot be only due to poor signal-to-noise ratios. The variability seen in the ULXs has been proposed to be associated with the high-energy components that may (extrinsically) vary due to the turbulences of the winds that, from time to time, encounter our line of sight (LOS; e.g., Middleton et al. 2015a). Observational evidence of winds in ULXs has been obtained from the detection of absorption lines with blueshifts of $\sim 0.2 c$ in high-quality XMMNewton/RGS spectra of some ULXs (e.g., Pinto et al. 2016), the discovery of the first extended X-ray bubble around the PULX NGC 5907 ULX-1 (Belfiore et al. 2020), and the optical bubbles around several ULXs (e.g., Pakull \& Mirioni 2002).

Furthermore, significant long-term (days to years) flux variability is observed in the vast majority of the ULXs for which multiple observations are available. However, only a small fraction of ULXs can be considered transient (i.e., with flux variation up to two orders of magnitude). Among the transient ULXs, the largest variations (up to a factor of 500) have been observed in the PULXs. This temporal behavior may
Table 1

Log of the XMM-Newton Observations

\begin{tabular}{|c|c|c|c|c|}
\hline No. & Obs.ID. & Date & $\begin{array}{l}\text { Instrument } \\
\text { EPIC-pn }\end{array}$ & Tot. Expos. \\
\hline 1 & 0303560701 & 2005 May 6 & $\mathrm{pn}+\mathrm{MOS}$ & $10.2 \mathrm{ks}$ \\
\hline 2 & 0824450401 & 2018 May 18 & $\mathrm{pn}+\mathrm{MOS}$ & $92.4 \mathrm{ks}$ \\
\hline
\end{tabular}

be explained by the onset/offset of the propeller mechanism (e.g., Tsygankov et al. 2016; Israel et al. 2017). Hence, the transient ULXs clearly play an important role in the ULX scenario, as they may be ideal places to look for new PULX candidates (e.g., Earnshaw et al. 2018; Song et al. 2019).

Here, we focus on the ULXs populating NGC 7456, a spiral galaxy at a distance of $\sim 15.7 \mathrm{Mpc}$ (Tully et al. 2016; Galactic column density of neutral hydrogen expected along the LOS of $8.7 \times 10^{19} \mathrm{~cm}^{-2}$, HI4PI Collaboration 2016), and hosting at least four ULXs (Walton et al. 2011). The galaxy was observed for the first time in the X-rays in 2005 by XMM-Newton, but the observation was short and strongly affected by high background, preventing any reliable investigation of the ULX properties. No other X-ray observations with XMM-Newton or other X-ray satellites have been performed since then, until, in 2018, our group obtained a new, deeper observation in the context of an XMM-Newton Large Programme (PI: G. Israel). This new data set allowed us to perform a more constraining characterization of the ULXs in the galaxy and the probable identification of a new ULX on its outskirts.

\section{Data Reduction}

NGC 7456 was observed twice by XMM-Newton, first in 2005 May (Obs.ID: 0303560701; PI: D. Rosa González) and then in 2018 May (Obs.ID: 0824450401; PI: G. Israel), with total exposure times of $\sim 10 \mathrm{ks}$ and $\sim 92 \mathrm{ks}$, respectively (Table 1). The observations were processed with the Scientific Analysis Software (SAS) v.16.1. We reduced the data from the EPIC cameras (all operated in full frame mode and with a thin filter), selecting events with FLAG $=0$, and PATTERN $\leqslant 4$ and PATTERN $\leqslant 12$ for $\mathrm{pn}$ and MOS, respectively. We filtered only the 2018 observation for high-background time intervals; the first observation was entirely taken during a highbackground period, hence we chose not to filter it for proton flares in order to avoid rejecting all events of this observation. The net exposures for the 2005 and 2018 observations were $\sim 10 \mathrm{ks}$ and $\sim 80 \mathrm{ks}$, respectively. For pn and MOS data, we extracted source and background events from circular regions of radii of $30^{\prime \prime}$ and $65^{\prime \prime}$, respectively. The photon times of arrival were converted to the solar system barycenter with the SAS task BARYCEN, using the best X-ray coordinates of each source reported in Walton et al. (2011).

All the spectra were rebinned with at least 25 counts per bin using the Ftool GRPPHA. In our spectral analysis, we fitted simultaneously the EPIC-pn and EPIC-MOS data, in the 0.3-10 keV range, with XSPEC v.12.10.1 (Arnaud 1996). In all fits, we included a multiplicative constant to take into account possible miscalibration of the relative flux between the three instruments. These did not vary for more than $10 \%$, as expected (Madsen et al. 2015).

We produced background-subtracted light curves in the $0.2-12 \mathrm{keV}$ energy band for all sources in the two observations 

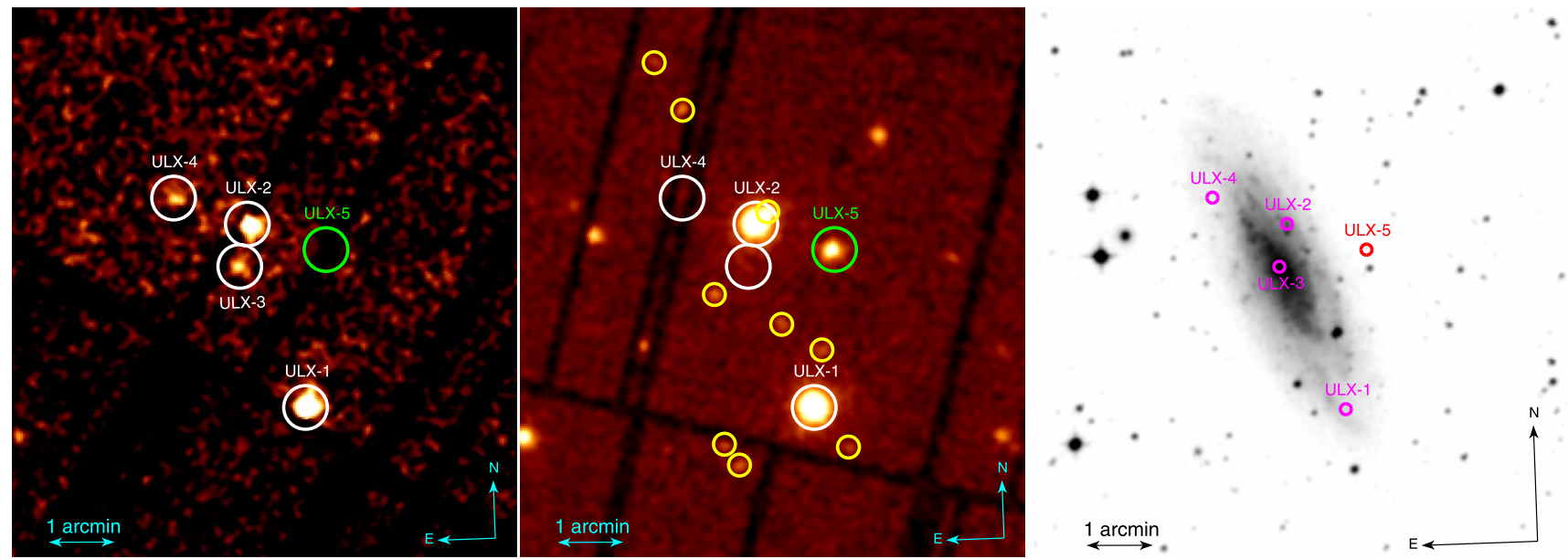

Figure 1. Comparison of the mosaics created by stacking cleaned EPIC-pn and MOS1-2 images in the 2005 (left) and 2018 (center) XMM-Newton observations. Four ULXs (ULX-1, ULX-2, ULX-3, and ULX-4; white circles) are observed during the 2005 observation, while ULX-3 and ULX-4 are not detected anymore in 2018 despite a much longer exposure time. A new source (tentatively labeled as ULX-5, green circle) is detected in the latest observation. We note that ULX-4 and ULX-5 were not on bad CCD stripes or gaps in the MOS images of 2005 and 2018. Other fainter sources were detected in the galaxy (yellow circles). Right: DSS optical image of the galaxy NGC 7456. The ULX positions are indicated with magenta circles (radius of $5^{\prime \prime}$ ), while the ULX-5 is indicated by a red circle.

using the EXTraS ${ }^{24}$ tools and prescriptions (De Luca et al. 2016 and De Luca et al. in preparation). Count rates of light curves from different EPIC cameras were converted into fluxes using the conversion factor in the Processing Pipeline Subsystem products files and then combined (Marelli et al. 2017) to obtain the total light curve.

\section{Data Analysis}

Walton et al. (2011) reported the presence of four ULXs in the galaxy NGC 7456 (Figure 1, left) using the 2005 XMMNewton observation. We carried out a source detection on the new 2018 XMM-Newton observation, applying the task EDETECT_CHAIN (setting a likelihood threshold limit to 15) to the combined EPIC-pn and EPIC-MOS images in the $0.2-12 \mathrm{keV}$ energy band. We found that only ULX-1 and ULX-2 were significantly detected. ULX-3 and ULX-4 were instead below the detection threshold, suggesting that they went back to quiescence. We also found a previously undetected source (at $\sim 78^{\prime \prime}$, i.e., $\sim 6 \mathrm{kpc}$ of projected distance, from the center of the galaxy) that we tentatively labeled as ULX-5 because it is bright and it lies inside the size of the galaxy NGC 7456 (Paturel et al. 2003).

In addition, superimposed to the galaxy, we detected at least nine other fainter X-ray sources (Table 2; yellow circles in Figure 1-center), with fluxes between $\sim(0.4-2) \times 10^{-14}$ erg $\mathrm{cm}^{-2} \mathrm{~s}^{-1}$. Assuming they are all hosted in NGC 7456 and have an absorbed power-law spectral shape with $n H=10^{21} \mathrm{~cm}^{-2}$ and $\Gamma=2$, their $0.3-10 \mathrm{keV}$ luminosities are $1-6 \times 10^{38} \mathrm{erg} \mathrm{s}^{-1}$. A fit with a constant model of their light curves binned with $\Delta T=10 \mathrm{ks}$ showed that, except for one, all the sources did not display significant flux variability during the observation, with a null hypothesis probability higher than 0.05 .

\footnotetext{
${ }^{24}$ http://www.extras-fp7.eu
}

Table 2

Positions of the Sources Found (at $>3 \sigma$ Significance) in the Galaxy NGC 7456 Using the 2018 XMM-Newton Observation

\begin{tabular}{lcccc}
\hline \hline Source & R.A. & Decl. & $\begin{array}{c}\text { Stat. err. } \\
\text { arcsec }\end{array}$ & $\begin{array}{c}\text { Rates }_{0.3-10 \mathrm{keV}} \\
\text { cts s}^{-1}\end{array}$ \\
\hline ULX-1 & $23: 02: 05.62$ & $-39: 36: 17.0$ & 0.1 & $0.159 \pm 0.002$ \\
ULX-2 & $23: 02: 09.73$ & $-39: 33: 26.9$ & 0.1 & $0.145 \pm 0.002$ \\
ULX-3 $^{\text {a }}$ & $23: 02: 10.64$ & $-39: 34: 04.7$ & & \\
ULX-4 $^{\text {a }}$ & $23: 02: 15.15$ & $-39: 33: 01.1$ & & \\
ULX-5 $^{23: 02: 03.80}$ & $-39: 33: 52.0$ & 0.2 & $0.0235 \pm 0.0008$ \\
\hline X6 & $23: 02: 08.86$ & $-39: 33: 15.2$ & 0.3 & $0.0119 \pm 0.0007$ \\
X7 & $23: 02: 15.30$ & $-39: 31: 39.1$ & 0.5 & $0.0052 \pm 0.0005$ \\
X8 & $23: 02: 13.33$ & $-39: 34: 29.0$ & 0.6 & $0.0039 \pm 0.0004$ \\
X9 & $23: 02: 11.85$ & $-39: 37: 06.1$ & 0.7 & $0.0036 \pm 0.0004$ \\
X10 & $23: 02: 17.41$ & $-39: 30: 54.6$ & 1.1 & $0.0029 \pm 0.0004$ \\
X11 & $23: 02: 04.98$ & $-39: 35: 23.4$ & 0.8 & $0.0029 \pm 0.0004$ \\
X12 & $23: 02: 08.09$ & $-39: 34: 58.6$ & 0.9 & $0.0028 \pm 0.0004)$ \\
X13 & $23: 02: 12.98$ & $-39: 36: 46.5$ & 0.9 & $0.0027 \pm 0.0004$ \\
X14 & $23: 02: 03.16$ & $-39: 36: 53.3$ & 1.0 & $0.0018 \pm 0.0003$ \\
\hline
\end{tabular}

Note. We report the source position, the statistical error, and the source count rate (pn plus $\mathrm{MOS} 1+2$ ).

a In 2018, these sources were not detected, hence for completeness we report the coordinates given in Walton et al. (2011).

\subsection{ULX-1}

\subsubsection{Spectral Analysis}

We fitted simultaneously the time-averaged EPIC-pn and MOS spectra of the 2018 observation. We first adopted a simple phenomenological model, i.e., an absorbed multi-color blackbody disk (DISKBB; Mitsuda et al. 1984) plus a blackbody (BBODYRAD in XSPEC), which, as in other ULXs, provided a good description of the $0.3-10 \mathrm{keV}$ spectra (e.g., Stobbart et al. 2006; Pintore et al. 2015; Rodríguez Castillo et al. 2019). As commonly found with ULXs, we note that other alternative two-component models as a DISKBB+NTHCOMP, a DISKBB + CUTOFFPL or a DISKBB + HIGHECUT $\times$ POWERLAW provide good fits as well.

The TBABS(DISKBB+BBODYRAD) model gave a good fit $\left(\chi^{2} /\right.$ dof $\left.\sim 371.7 / 354\right)$; we report the best-fit parameters in 
Table 3

Best-fit Spectral Parameters of the ULXs Detected in the XMM-Newton Observations

\begin{tabular}{|c|c|c|c|c|c|c|c|c|c|c|c|c|c|}
\hline src. & year & $\begin{array}{r}n H \\
10^{22} \mathrm{~cm}^{-2}\end{array}$ & $\begin{array}{r}k T_{\mathrm{dbb}} \\
\mathrm{keV}\end{array}$ & $p$ & Norm. & $k T_{\mathrm{bb}} / \Gamma$ & Norm. & $\begin{array}{l}E_{\text {line }} \\
\mathrm{keV}\end{array}$ & $\begin{array}{l}\sigma_{\text {line }} \\
\mathrm{keV}\end{array}$ & $\begin{array}{l}N_{\text {line }} \\
\mathrm{keV}\end{array}$ & $\begin{array}{c}\text { Flux }^{\mathrm{a}} \\
10^{-13} \mathrm{erg} \mathrm{cm}^{-2} \mathrm{~s}^{-1}\end{array}$ & $\begin{array}{c}\mathrm{L}_{X} \\
10^{39} \mathrm{erg} \mathrm{s}^{-1}\end{array}$ & $\chi_{\nu}^{2} / \mathrm{dof}$ \\
\hline ULX-1 & $\begin{array}{l}2005 \\
2018\end{array}$ & $\begin{array}{r}0.049 \text { (fixed) } \\
0.05_{-0.01}^{+0.02} \\
0.09_{-0.03}^{+0.03}\end{array}$ & $\begin{array}{l}0.29_{-0.04}^{+0.04} \\
0.27_{-0.02}^{+0.02} \\
0.23_{-0.03}^{+0.02}\end{array}$ & $\begin{array}{l}\cdots \\
\cdots \\
\cdots\end{array}$ & $\begin{array}{l}2.0_{-0.9}^{+1.8} \\
1.8_{-0.5}^{+0.7} \\
5.3_{-2.4}^{+7.6}\end{array}$ & $\begin{array}{c}1.0_{-0.4}^{+0.7} \mathrm{keV} \\
0.80_{-0.07}^{+0.09} \mathrm{keV} \\
0.73_{-0.07}^{+0.08} \mathrm{keV}\end{array}$ & $\begin{array}{l}0.012_{-0.009}^{+0.05} \\
0.012_{-0.004}^{+0.006} \\
0.019_{-0.007}^{+0.01}\end{array}$ & $\begin{array}{c}\cdots \\
\cdots \\
0.67_{-0.03}^{+0.03}\end{array}$ & $\begin{array}{c}\cdots \\
\cdots \\
0.09_{-0.05}^{+0.06}\end{array}$ & $\begin{array}{c}\cdots \\
\cdots \\
0.07_{-0.04}^{+0.1}\end{array}$ & $\begin{array}{c}2.9 \pm 0.5 \\
1.65 \pm 0.05 \\
1.63 \pm 0.05\end{array}$ & $\begin{aligned} 10.0 & \pm 1.0 \\
5.9 & \pm 0.4 \\
6.9 & \pm 0.6\end{aligned}$ & $\begin{array}{c}1.00 / 84 \\
1.05 / 354 \\
0.97 / 351\end{array}$ \\
\hline ULX-2 & 2005 & $\begin{array}{r}0.4_{-0.2}^{+0.2} \\
0.08_{-0.08}^{+0.2} \\
0.24_{-0.04}^{+0.05} \\
0.35_{-0.03}^{+0.02}\end{array}$ & $\begin{array}{c}\cdots \\
1.3_{-0.3}^{+0.3} \\
0.56_{-0.09}^{+0.1} \\
3.1_{-0.5}^{+0.7}\end{array}$ & $\begin{array}{l}\ldots \\
\ldots\end{array}$ & $\begin{array}{c}4_{-2}^{+5} \times 10^{-5} \\
0.09_{-0.04}^{+0.09} \\
\left(2.7_{-1.4}^{+3.5}\right) \times 10^{-5}\end{array}$ & $\begin{array}{c}2.0_{-0.3}^{+0.4} \\
\ldots \\
1.3_{-0.2}^{+0.4} \mathrm{keV} \\
\ldots\end{array}$ & $\begin{array}{c}\left(6.4_{-2.2}^{+3.2}\right) \times 10^{-5} \\
\ldots \\
\left(4.2_{-2.6}^{+4.3}\right) \times 10^{-3} \\
\ldots\end{array}$ & $\begin{array}{l}\cdots \\
\cdots \\
\cdots \\
\cdots\end{array}$ & $\begin{array}{l}\cdots \\
\cdots \\
\cdots \\
\cdots\end{array}$ & $\begin{array}{l}\cdots \\
\cdots \\
\cdots \\
\cdots\end{array}$ & $\begin{array}{c}2.3_{-0.7}^{+0.6} \\
2.3_{-0.7}^{+0.6} \\
2.3 \pm 0.1 \\
2.4 \pm 0.1\end{array}$ & $\begin{array}{l}6.9 \pm 0.2 \\
6.9 \pm 0.2 \\
8.4 \pm 0.2 \\
9.9 \pm 0.2\end{array}$ & $\begin{array}{c}1.33 / 32 \\
1.20 / 32 \\
1.07 / 311 \\
1.04 / 312\end{array}$ \\
\hline ULX-5 & 2018 & $0.04_{-0.03}^{+0.04}$ & $\ldots$ & $\ldots$ & $\ldots$ & $1.9_{-0.2}^{+0.2}$ & $7.0_{-0.8}^{+1.0}$ & $\ldots$ & $\ldots$ & $\ldots$ & $0.40_{-0.05}^{+0.05}$ & $1.3 \pm 0.1$ & $0.90 / 81$ \\
\hline
\end{tabular}

Notes. Errors are at the $90 \%$ confidence level for each parameter of interest.

${ }^{a}$ EPIC absorbed flux in the $0.3-10 \mathrm{keV}$ energy band. 
ULX-1

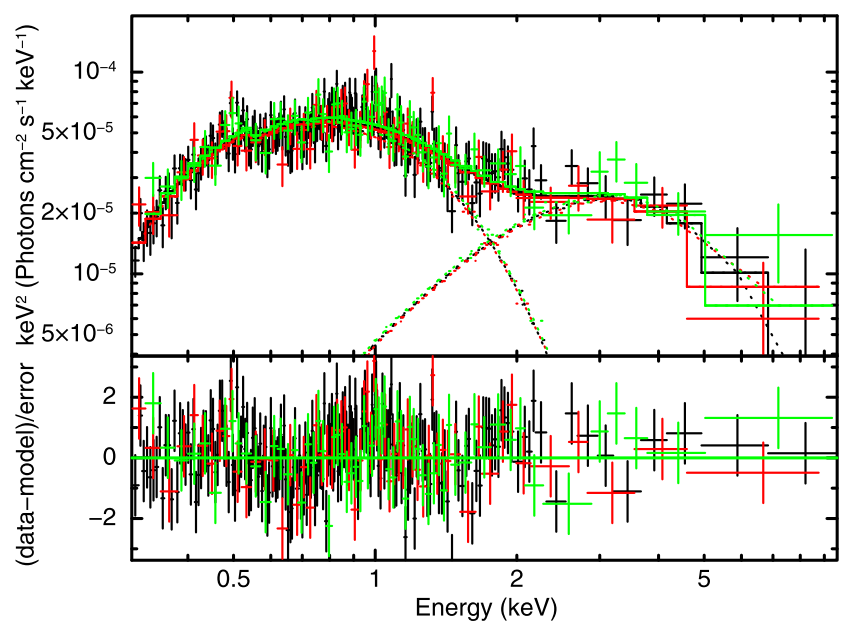

ULX-2

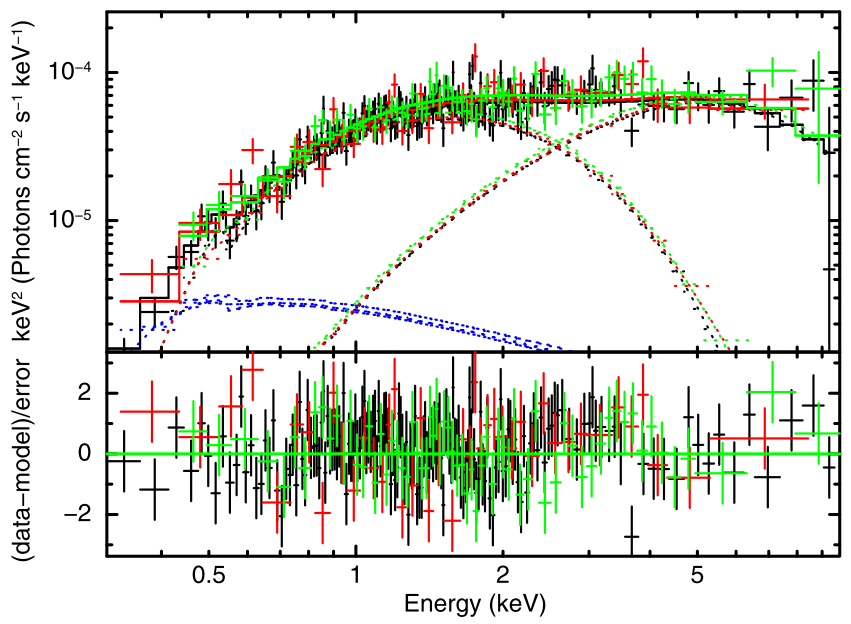

ULX-5

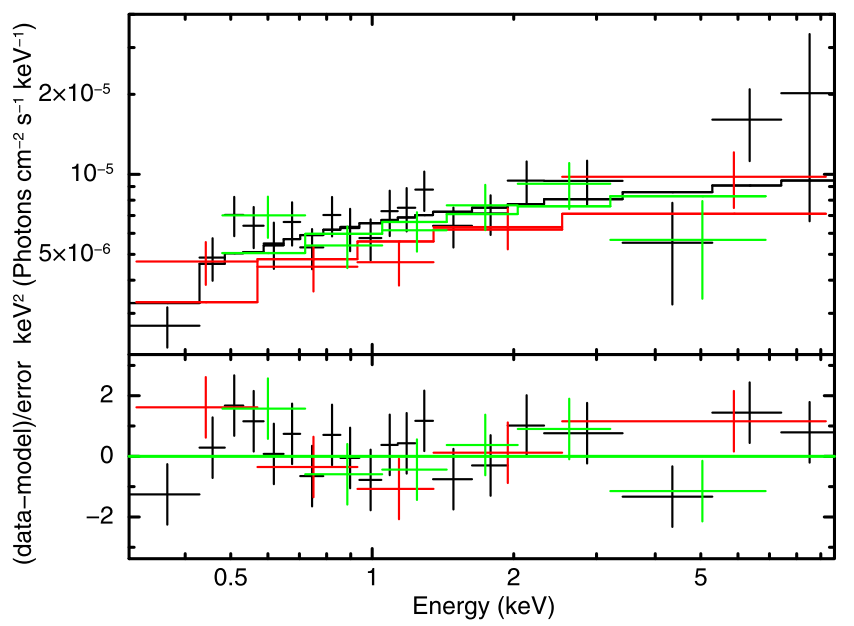

Figure 2. Top: unfolded $\left(E^{2} \mathrm{f}(E)\right)$ spectra of ULX-1 (TBABS(DISKBB+BBODYRAD), left), ULX-2 (TBABS(DISKBB+BBODYRAD), center), and ULX-5 (TBABS (POWERLAW), right). EPIC-pn spectra are indicated with black points, while EPIC-MOS1 and 2 are in red and green, respectively. The solid lines represent the best-fit models, while in the bottom panels the corresponding residuals are shown. In the central plot, we also show in blue dashed lines the spectrum of the contaminant source (see Section 3.2). All spectra have been further rebinned for display purposes.

Table 3. We found a column density $\left(\sim 5 \times 10^{20} \mathrm{~cm}^{-2}\right)$ higher than the Galactic one, and temperatures of $k T_{\mathrm{dbb}} \sim 0.27 \mathrm{keV}$ and $k T_{\mathrm{bb}} \sim 0.8 \mathrm{keV}$. The latter are associated with emitting radii of $\sim 2000-7000 \mathrm{~km}$ (assuming an unknown inclination angle between $5^{\circ}$ and $85^{\circ}$ and no color-correction factor) and $170 \pm 35 \mathrm{~km}$ for the DISKBB and BBODYRAD models, respectively. ULX-1 was quite soft (Figure 2, left), with an unabsorbed $0.3-10 \mathrm{keV}$ flux of $(2.0 \pm 0.1) \times 10^{-13} \mathrm{erg} \mathrm{cm}^{-2}$ $\mathrm{s}^{-1}$, implying an unabsorbed luminosity of $(6.0 \pm 0.4) \times 10^{39} \mathrm{erg} \mathrm{s}^{-1}$.

We note that, in the case of super-Eddington accretion, modeling the accretion disk with an optically thick and geometrically thin disk may not be appropriate. In such a scenario, the disk should instead be modeled with a "slim" disk (i.e., a geometrically and optically thick disk). Some authors (e.g., Walton et al. 2018) showed that a possible description of the ULX spectra is given by the combination of DISKBB and DISKPBB models (where, in the latter, the radial dependence of the temperature is given by $r^{-p}$, and $p$ is 0.75 for a standard disk and 0.5 for an advection-dominated disk). Thus, we tentatively substituted first the DISKBB with a DISKPBB model: this model was suitable for the data as well, although we found that $p$ was unconstrained. Second, we also substituted the highenergy BBODYRAD with the DISKPBB model: even in this case, although the fit was formally good, the $p$ parameter was not constrained. This implies that the current data quality do not allow us to rule out the existence of a thick disk yet in ULX-1.

Hereafter, we will consider only the DISKBB + BBODYRAD model results. Although the best-fit with this model is statistically acceptable, some residuals in absorption around $0.6-0.7 \mathrm{keV}$ (or, alternatively, in emission around $1 \mathrm{keV}$ ) are still observed (see Figure 2-left). These are often found in the ULX spectra (see e.g., Middleton et al. 2015b). We can fit the residual in absorption with a Gaussian absorption line (GABS in XSPEC), obtaining an improvement of $\Delta \chi^{2}=33$ for three additional d.o.f. The inclusion of this feature slightly changed the continuum spectral parameters, giving $n \mathrm{H}$ $=(9 \pm 3) \times 10^{20} \mathrm{~cm}^{-2}, \quad k T_{\mathrm{dbb}}=0.23_{-0.03}^{+0.02} \mathrm{keV} \quad$ and $k T_{\mathrm{bb}}=0.73_{-0.07}^{+0.08} \mathrm{keV}$. The line energy, its FWHM $(\sigma)$, and the line depth (i.e., the equivalent width) converged to $E=0.67 \pm 0.03 \mathrm{keV}, \sigma=0.09_{-0.05}^{+0.06} \mathrm{keV}$, and $0.07_{-0.04}^{+0.1} \mathrm{keV}$, respectively. We do not consider this feature an artifact of the modeling because the two spectral components intersect around $1.5-2 \mathrm{keV}$, i.e., well above the line energy. Hence, should the 

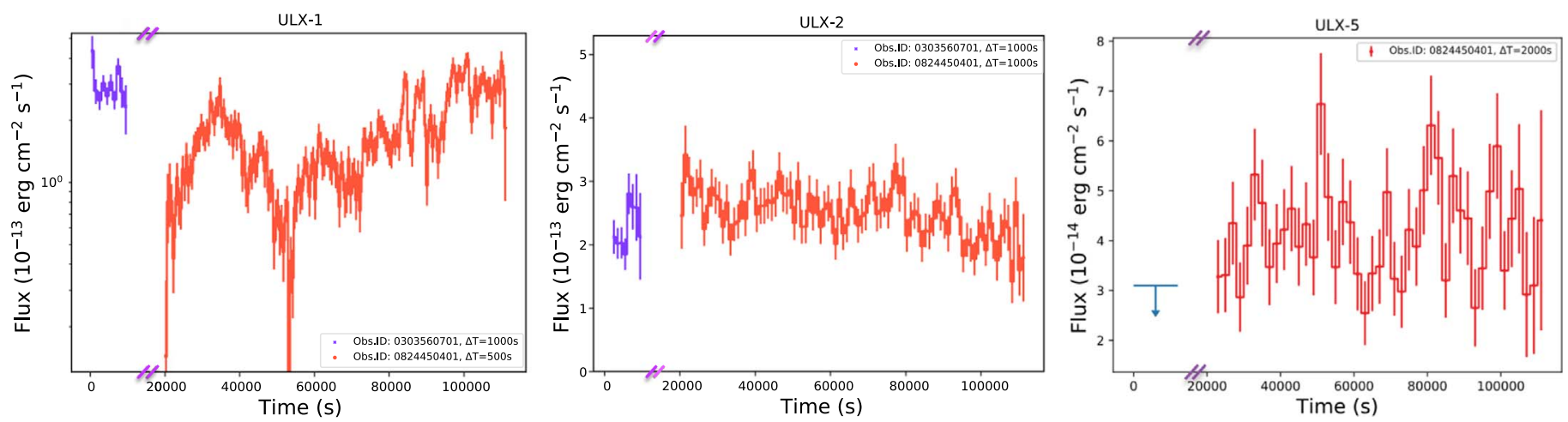

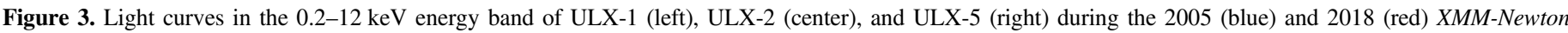
observations. The $X$-axis is arbitrary, as the gap within the two observations was shortened for display purposes.

feature be real, it might be associated with a blending of ionized oxygen lines in absorption (O VII-VIII at $\sim 0.5-0.7 \mathrm{keV}$ ). ULX-1 was not in the field of view of the RGS instrument in 2018 and its flux was too low for the RGS instruments, therefore no high-resolution X-ray spectra are currently available to further investigate the nature of such a possible feature.

Finally, we note that the TBABS(DISKBB+BBODYRAD) model is adequate for the 2005 data as well, although we had to fix the absorption to the 2018 best-fit $n H\left(4.9 \times 10^{20} \mathrm{~cm}^{-2}\right)$, as this is otherwise unconstrained. The other best-fit parameters are shown in Table 3 and they are consistent with those of 2018 to within uncertainties. On the other hand, we remark that these results have to be considered with caution, as the observation was fully affected by high background. We measured an unabsorbed $0.3-10 \mathrm{keV}$ flux of $(3.4 \pm 0.5) \times 10^{-13} \mathrm{erg} \mathrm{cm}^{-2}$ $\mathrm{s}^{-1}$ corresponding to a luminosity of $(1.0 \pm 0.1) \times 10^{40} \mathrm{erg} \mathrm{s}^{-1}$ (in agreement with Walton et al. 2011), a factor of $\sim 2$ higher than the average flux in 2018.

\subsubsection{Temporal Properties}

In 2005, ULX-1 was the brightest source in NGC 7456 at a luminosity of $\sim 10^{40} \mathrm{erg} \mathrm{s}^{-1}$ (Walton et al. 2011). Because of the short exposure time and high-background level, its 0.3-10 keV XMM-Newton light curve accumulated in bins of $500 \mathrm{~s}$ (Figure 3 left) gives little information on the source variability.

Instead, the longer 2018 XMM-Newton observation allowed us to find a highly significant variability with recurrent increments of flux up to an order of magnitude (see Figure 3-left), on timescales of only a few kiloseconds. We found that the $0.3-10 \mathrm{keV}$ fractional variability $\mathrm{F}_{\mathrm{var}}$ (e.g., Vaughan et al. 2003), on timescales $>500 \mathrm{~s}$, is $47 \% \pm 1 \%$ : this is one of the highest non-periodic short-term variabilities presently measured in a ULX (e.g., Sutton et al. 2013; Middleton et al. 2015a). We verified that such a high variability is also preserved on smaller timescales $(<500 \mathrm{~s})$.

To better assess the origin of such a variability, we evaluated the hardness ratios of the net counts between the $0.3-1.0 \mathrm{keV}$ and $1.0-10 \mathrm{keV}$ energy bands, chosen such that the number of counts in each band is comparable. The hard-to-soft band ratio $(\mathrm{H} / \mathrm{S})$ clearly shows a variability during the $80 \mathrm{ks}$ of observation, in particular after the first $\sim 25 \mathrm{ks}$ of the observation where there is an indication of a softening that apparently tracks periods of very low fluxes (Figure 4-top).
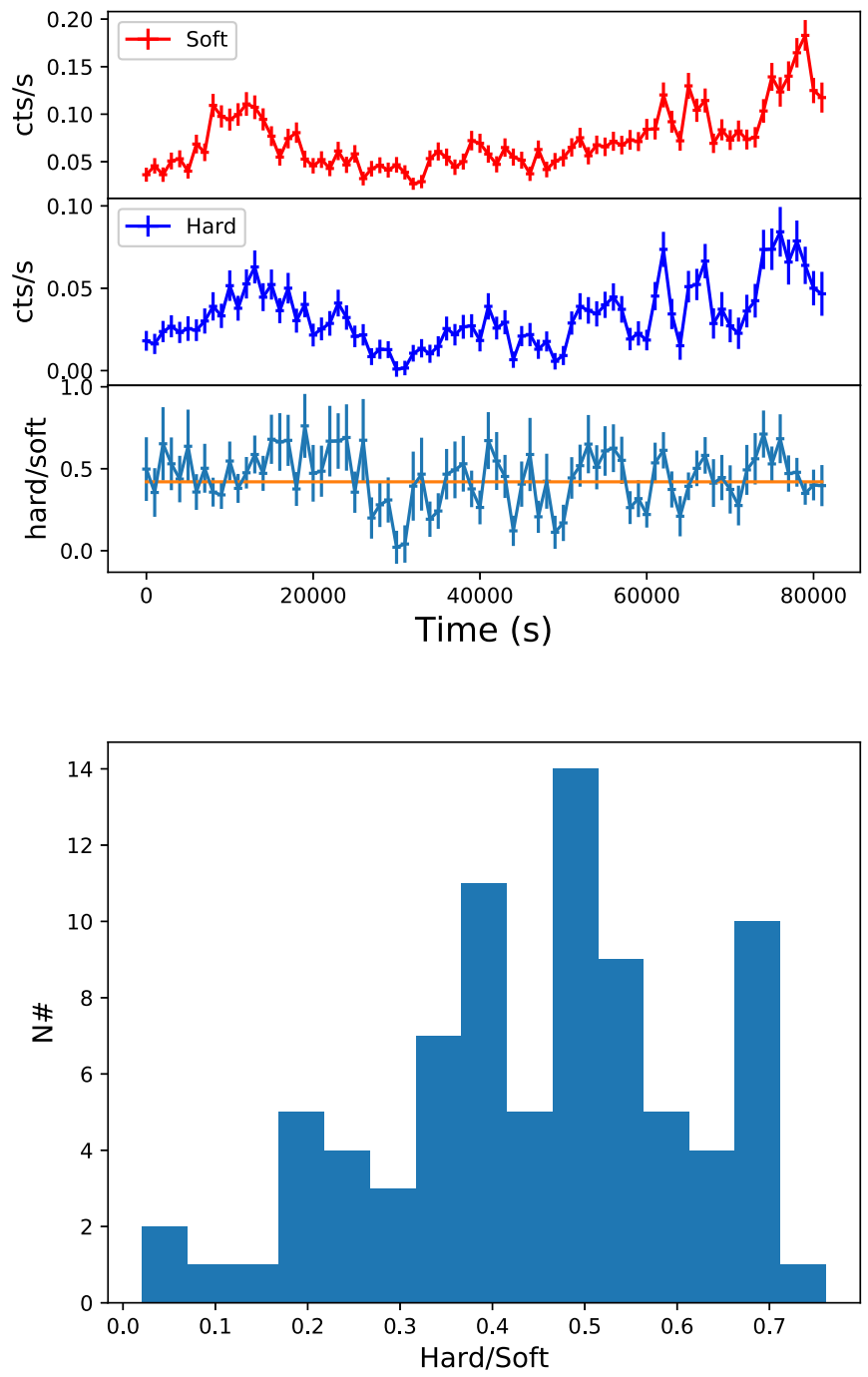

Figure 4. Top: EPIC-pn soft (0.3-1.0 keV, top panel) and hard (1.0-10 keV, center panel) background-subtracted light curves of ULX-1 $(\Delta T=1000 \mathrm{~s})$ compared with their hardness ratio (bottom panel), during the $2018 X M M$ Newton observation. The orange line is the best fit $(\mathrm{H} / \mathrm{S}=0.42)$ with a constant model. Bottom: distribution of the hardness ratios that indicates how the spectral variability is not normally distributed.

Finally, we also searched for coherent pulsations in the 2018 data. Adopting a generalization of the Fourier-based procedure described in Israel \& Stella (1996; see also Rodríguez Castillo et al. 2019), we could only place $3 \sigma$ upper limits on the pulse 


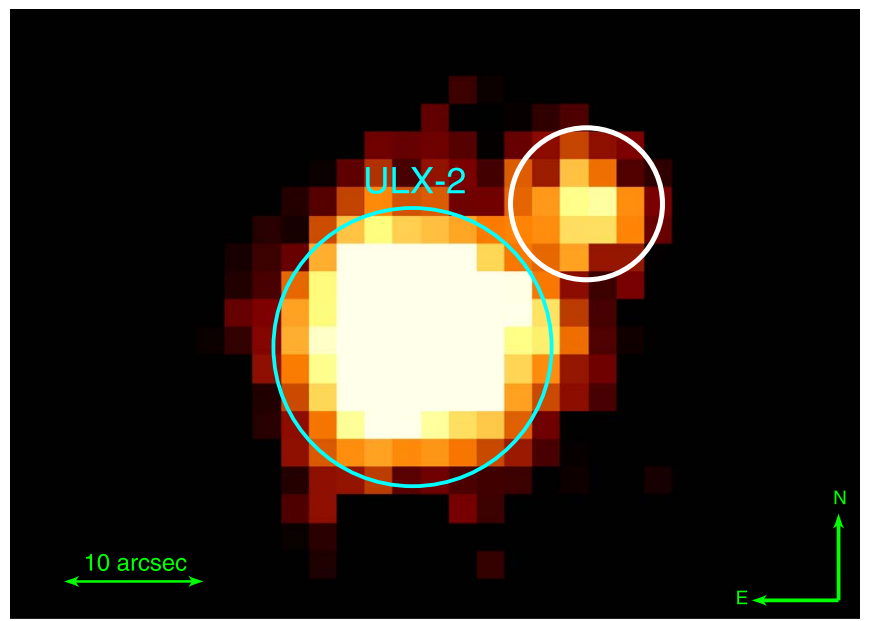

Figure 5. 2018 EPIC image of the field of ULX-2 (cyan circle). A weaker and soft source (white circle) lies at $\sim 15^{\prime \prime}$ to ULX-2.

fraction of $17 \%-19 \%$ for periods in the range $150 \mathrm{~ms}-500 \mathrm{~s}$, assuming a sinusoidal pulse profile. This limit is, on average, slightly larger than the pulse fraction observed in PULXs.

\subsubsection{Hardness-resolved Spectroscopy}

In this section, we investigate in greater detail the spectral variability of the 2018 observation suggested by the hardness ratios. Here we perform hardness-resolved spectroscopy to track or identify specific patterns with the ULX spectral state. The bottom panel of Figure 4 suggests the existence of at least two different spectral states, approximately above and below $\mathrm{H} / \mathrm{S}=0.42$. We selected this threshold to extract two EPIC spectra for both states, and we fitted them simultaneously, adopting the same model of the averaged spectrum (i.e., tbabs $($ diskbb+bbodyrad)). As a first step, we left all the parameters free to vary independently. We found that the normalizations of the soft component and the temperatures of the two thermal components were consistent, to within uncertainties, between the two spectra; for this reason we kept them linked. Instead, the hottest blackbody normalizations were different for the two spectra, indicating that the flux variations are mostly driven by the high-energy component. This fit gave $\chi^{2} /$ dof $=420 / 352$.

However, several residuals around $0.7 \mathrm{keV}$ were still present, as already seen for the average spectrum. Also, in this case we added a GABS model, fixing the feature energy and width $(0.67$ and $0.09 \mathrm{keV})$ to the best-fit values found for the average spectrum. The final best fit $\left(\chi^{2} /\right.$ dof $\left.=389.15 / 350\right)$ provided the following parameters: $n H=9_{-2}^{+3} \times 10^{20} \mathrm{~cm}^{-2}$, $k T_{\mathrm{dbb}}=0.22_{-0.02}^{+0.02} \mathrm{keV}$, and $k T_{\mathrm{bb}}=0.68_{-0.05}^{+0.06} \mathrm{keV}$. These are slightly different from those inferred in the average spectrum. The blackbody normalizations for the low- and high-H/S spectra were $0.015_{-0.005}^{+0.008} \mathrm{ph} \mathrm{cm}^{-2} \mathrm{~s}^{-1}$ and $0.032_{-0.01}^{+0.01} \mathrm{ph} \mathrm{cm}^{-2}$ $\mathrm{s}^{-1}$, respectively, implying that the emitting radii varied from $\sim 200$ to $\sim 300 \mathrm{~km}$. The depth of GABS is instead consistent between low and high states at a value of $0.07_{+0.02}^{-0.02} \mathrm{keV}$.

\section{2. $U L X-2$}

The deep 2018 XMM-Newton observation allowed us to find that ULX-2 lies close to another source at $\sim 15^{\prime \prime}$ (see Figure 5). This implies that the contamination from the latter can only be partially removed. Through a maximum likelihood analysis based on the EPIC-pn/MOS point-spread functions (see the approach in Rigoselli \& Mereghetti 2018), we extracted the spectrum of the second source and we estimated that it is very soft (most of the photons are below $2 \mathrm{keV}$ ). It can be modeled $\left(\chi_{\nu}^{2}<1\right)$ by a single absorbed power law with $\Gamma=3.3_{-1.2}^{+2.4}$ and $n H=\left(1.9_{-1.9}^{+0.4}\right) \times 10^{21} \mathrm{~cm}^{-2}$. We estimated an absorbed $0.3-10 \mathrm{keV}$ flux of $\left(8_{-3}^{+5}\right) \times 10^{-15} \mathrm{erg} \mathrm{cm}^{-2} \mathrm{~s}^{-1}$. Assuming the source is in NGC 7456, this corresponds to a luminosity of $\sim 2 \times 10^{38} \mathrm{erg} \mathrm{s}^{-1}$, which is well below the ULX luminosity threshold and consistent with the Eddington limit of an NS. We included the spectral model of the contaminants in the fit of the ULX-2 spectra.

\subsubsection{Temporal Analysis}

In the 2005 observation, ULX-2 was the second most luminous ULX in the galaxy and apparently at a constant flux level during the observation. The 2018 source light curve instead showed a limited short-term variability, overimposed on a general decay trend during the observation (Figure 3-center). We modeled the light curve with a constant and it does not fit the data well (null hypothesis probability $=$ $1.1 \times 10^{-5}$ ). No coherent pulsations were detected, with a $3 \sigma$ upper limit of $17 \%-19 \%$ for periods in the range $150 \mathrm{~ms}-500 \mathrm{~s}$. This is similar to ULX-1 because they have comparable statistics.

\subsubsection{Spectral Analysis}

We fitted the 2018 average EPIC-pn and MOS spectra of ULX-2 with an absorbed DISKBB +BBBODYRAD model that provided a very good fit ( $\chi^{2}=332.8$ for 311 dof; see Table 3 ). The emitting radii for the two components were $400-1400 \mathrm{~km}$ (assuming an inclination angle between $5^{\circ}$ and $85^{\circ}$ and no color-correction factor) and $102_{-40}^{+45} \mathrm{~km}$. We estimated an unabsorbed $0.3-10 \mathrm{keV}$ flux of $(2.8 \pm 0.1) \times 10^{-13} \mathrm{erg} \mathrm{cm}^{-2}$ $\mathrm{s}^{-1}$, corresponding to a luminosity of $(8.4 \pm 0.2) \times 10^{39} \mathrm{erg} \mathrm{s}^{-1}$. Hence this was the most luminous ULX in the galaxy during the observation.

We also tested a thick disk scenario, fitting the spectra with a single DISKPBB model and finding that it fits the data very well $\left(\chi^{2} /\right.$ dof $\left.=324.41 / 312\right)$. The model shows some evidence for a thick disk $(p \sim 0.5)$, with an inner temperature of $\sim 3 \mathrm{keV}$ (see Table 3 ). We also added a DISKBB to the DISKPBB model, to describe the low energy part of the spectrum. The improvement given by its inclusion was not statistically significant, however ( $\Delta \chi^{2} \sim 3$, for 2 additional dof).

In 2005 , the signal-to-noise ratio was poor ( $\sim 940$ net counts) and the data were fully affected by a high background. Fits of a single DISKBB or power law can provide good results (see Table 3). We tried also to fit the spectrum with the 2018 best-fit model, leaving only the DISKBB and BBODY normalizations free to vary. This model was, as expected, acceptable, with best-fit normalizations consistent with the previous values. From those values, we estimated an unabsorbed $0.3-10 \mathrm{keV}$ flux of $(3.0 \pm 1.0) \times 10^{-13} \mathrm{erg} \mathrm{cm}^{-2} \mathrm{~s}^{-1}$ and a luminosity of $(9 \pm 3) \times 10^{39} \mathrm{erg} \mathrm{s}^{-1}$.

\section{3. $U L X-3$ and $U L X-4$}

ULX-3 and ULX-4 were detected only in the 2005 observation with a luminosity of $\sim 9.2 \times 10^{38} \mathrm{erg} \mathrm{s}^{-1}$ and $\sim 1.2 \times 10^{39} \mathrm{erg} \mathrm{s}^{-1}$, respectively (Walton et al. 2011). In the 2018 observation, they were below the detection threshold and we estimated a $3 \sigma$ upper limit on their $0.3-10 \mathrm{keV}$ flux of 
$3.7 \times 10^{-15} \mathrm{erg} \mathrm{cm}^{-2} \mathrm{~s}^{-1}$, corresponding to a luminosity of $\sim 1.3 \times 10^{38} \mathrm{erg} \mathrm{s}^{-1}$, i.e., well below the ULX threshold, and implying a factor $\geqslant 10$ variability on long timescales.

\section{4. $U L X-5$}

During the 2005 observation, ULX-5 was undetectable and we estimated a $3 \sigma$ upper limit on the $0.3-10 \mathrm{keV}$ flux of $3.1 \times 10^{-14} \mathrm{erg} \mathrm{cm}^{-2} \mathrm{~s}^{-1}$, corresponding to a luminosity of $\sim 1.1 \times 10^{39} \mathrm{erg} \mathrm{s}^{-1}$. The 2018 source light curve is shown in the right panel of Figure 3 and no significant intra-observation variability was observed. Its spectrum was hard and could be modeled with a single absorbed power law, with a photon index of 1.9 (Figure 2-right and Table 3). The measured column density is higher than the Galactic one. The $0.3-10 \mathrm{keV}$ luminosity was $(1.3 \pm 0.1) \times 10^{39} \mathrm{erg} \mathrm{s}^{-1}$.

The hard spectrum and lack of intra-observation variability make the association with a foreground flaring star unlikely (e.g., Pye et al. 2015). Furthermore, in ESO/EFOSC2 observations in the $B$ band $(5 \times 180 \mathrm{~s})$ and $R$ band $(5 \times 300 \mathrm{~s})$ we did not find any optical counterpart down to $B \sim 22 \mathrm{mag}$ and $R \sim 23 \mathrm{mag}$. However, Wide-field Infrared Survey Explorer (WISE) detected within 2" a source with a W1 magnitude of $17.4 \pm 0.2 \mathrm{mag}$.

A Galactic X-ray source, assuming a reasonable distance of about $5 \mathrm{kpc}$, would have had a $0.3-10 \mathrm{keV}$ luminosity of $\sim 2 \times 10^{32} \mathrm{erg} \mathrm{s}^{-1}$. Should it be a magnetar, such a luminosity would be expected only in late stages of an outburst (see e.g Rea \& Esposito 2011; Coti Zelati et al. 2018). However, the sky position of the source, with a Galactic latitude $l \sim-34^{\circ}$, is not likely, given the spatial distribution of magnetars in our Galaxy (Olausen \& Kaspi 2014). A long-lasting Galactic accreting binary system outburst is unlikely as well, because we would expect to observe an optical counterpart (not detected). On the other hand, a low mass X-ray binary in quiescence cannot be excluded (e.g., Plotkin et al. 2013).

Because of its position with respect to the galaxy and its hard spectrum, we cannot rule out that it is a background active galactic nucleus (AGN). In fact, the upper limit to the opticalto-X-ray flux ratio of $\leqslant 0.8$ is consistent with those typical for AGNs and blazars (see, e.g., Maccacaro et al. 1988). Furthermore, from the $\log N$-Log $S$ of extragalactic sources (Moretti et al. 2003), we could estimate that $\sim 100$ objects are expected to be found in a square degree at the observed flux of ULX-5. Hence, for a galaxy dimension of 5 . $01 \times 1$ '.78, we estimated a $\sim 0.2$ background AGN in the field. Although we propose this source as a likely ULX candidate located in the outskirts of NGC 7456, deeper multiwavelength observations are needed to test its ULX nature and rule out a background AGN.

\section{Discussion}

In this work, we have carried out the first, detailed investigation of the properties of several ULXs hosted in the galaxy NGC 7456, thanks to a long-exposure XMM-Newton observation taken in 2018 (the only X-ray observation apart from a much shorter XMM-Newton observation taken in 2005). Two sources, ULX-1 and ULX-2, are very bright, with luminosities close to $10^{40} \mathrm{erg} \mathrm{s}^{-1}$, and were active during the two XMM-Newton observations. On the other hand, two ULXs in the galaxy (ULX-3 and ULX-4) may be considered candidate transient objects because they were not detected anymore in 2018 (although further monitoring is necessary to confirm their nature). Moreover, a possible new ULX candidate, with a luminosity slightly higher than $10^{39} \mathrm{erg} \mathrm{s}^{-1}$, was discovered in the 2018 data.

Unfortunately, no Hubble Space Telescope observations of the galaxy at the position of the ULXs have been taken, therefore the optical data are of quite poor resolution and little can be inferred about the ULX counterparts. We could associate ULX-1, ULX-2, and ULX-4 with the spiral arms of NGC 7456, while ULX-3 is close to the galactic center. We searched multiwavelength archives and found only WISE and Galaxy Evolution Explorer observations: their resolution is not adequate, however, to distinguish the emission from the singleULX counterparts from the faint diffuse, unresolved emission from the galaxy. For these reasons, no evidence of bubbles (or nebulae) can be inferred. Deeper observations are necessary to further investigate the nature of the ULX counterparts.

\section{1. $U L X-1$}

ULX-1 is a bright source (already reported in Walton et al. 2011) located in a spiral arm, at $\sim 2$ !3 from the center of NGC 7456. The archival optical/infrared images are not deep enough to allow us to find evidence of a clear counterpart. We only estimated a $5 \sigma$ upper limit on the magnitudes $M_{J} \geqslant 20.2$ and $M_{k_{s}} \geqslant 18.1 \mathrm{mag}$ (from the Vista Hemisphere Survey; McMahon et al. 2013). Our analysis showed that ULX1 is characterized by pronounced short-term variability on timescales down to $500 \mathrm{~s}$.

The $0.3-10 \mathrm{keV}$ source luminosity varied across the 2005 and 2018 observations in the range $(6-10) \times 10^{39} \mathrm{erg} \mathrm{s}^{-1}$. The high-quality 2018 ULX-1 spectrum showed that the source was quite soft (the ratio of the $1-10 \mathrm{keV}$ and $0.3-1 \mathrm{keV}$ fluxes was $\sim 30 \%$ ) and we found that its spectral shape can be well modeled with the combination of two thermal components. Because of its spectral and temporal properties, we can claim that the source was not in the typical hard or soft states of the Galactic accreting BHs in outburst (e.g., McClintock \& Remillard 2006; Belloni et al. 2011), hence we can consider the scenario of sub-Eddington accretion onto a massive $\mathrm{BH}$ of 40-100 $M_{\odot}$ less likely.

If the source is accreting at super-Eddington rates, the compact object might be surrounded by an accretion disk, or an extended outflow, with an inner radius of $2000-7000 \mathrm{~km}$. Outflows are expected in the case of super-Eddington accretion (Poutanen et al. 2007). These may be identified with the discovery of blueshifted absorption and emission lines (e.g., Pinto et al. 2016; Kosec et al. 2018) or X-ray bubble nebulae around ULXs (Belfiore et al. 2020). In our analysis, we report on a possible absorption feature at $\sim 0.7 \mathrm{keV}$ that is often observed in ULX spectra (e.g., Middleton et al. 2015b). The feature may then be associated with a blending of ionized oxygen features (O VII-O VIII).

However, the line might also be interpreted as a cyclotron line in an accreting NS scenario; this has been proposed for the source M51 ULX-8 as a candidate NS because of the first detection of a cyclotron absorption feature (Brightman et al. 2018; Middleton et al. 2019). The feature in ULX-1 might correspond to an NS magnetic field of $B_{12}=(1+z)$ $E_{c} / 11.6 \mathrm{keV} \sim 8 \times 10^{11} \quad \mathrm{G}$ and $B_{15}=(1+z) E_{c} / 6.3 \mathrm{keV} \sim 1.5 \times 10^{14} \mathrm{G}$ for an electron or proton feature, respectively, where $z$ is the gravitational redshift assumed to be 0.3 for an $\mathrm{NS}, E_{c}$ is the line energy 
$(0.67 \mathrm{keV})$, and $B_{12}$ and $B_{15}$ are the magnetic fields in units of $10^{12} \mathrm{G}$ and $10^{15} \mathrm{G}$, respectively.

According to Sutton et al. (2013), ULX-1 can be classified as a soft-ultraluminous source. It has been shown that the sources of this class are generally those with the highest short-term flux variability. This is confirmed by the 2018 data of ULX-1, in which there was a strong flux variability described by an overall flux increment imposed on short-term variability on timescales from hundreds to thousands of seconds $\left(F_{\text {var }} \sim 50 \%\right)$. This is among the highest variability ever observed in a ULX (e.g., Sutton et al. 2013). The source light curve is characterised by flux variations of more than an order of magnitude in $\sim 80 \mathrm{ks}$ with indications of possible flares or dips.

The flux evolution is also accompanied by spectral changes, although the interpretation is not straightforward. We found that the variability is mainly driven by the hard component, as already reported for the variability of soft-ultraluminous ULXs (e.g., Middleton et al. 2015a). The time evolution of the hardness ratios suggests that the spectral softening detected during low flux periods might be due to a reduction of the hard component emission. Such a result rules out a dipping activity caused by photoelectric absorption, as in such a case a hardening rather than a softening of the X-ray spectrum would be expected. However, the ULX-1 properties are similar to those observed in NGC 6946 ULX-3 (Earnshaw et al. 2019), NGC 253 ULX-1 (Barnard 2010), NGC 5907 ULX-1, and NGC 55 ULX-1 (although this is markedly fainter- $\sim 10^{39} \mathrm{erg} \mathrm{s}^{-1}$-and shows well defined dips on timescales of hundreds of seconds; e.g., Stobbart et al. 2004), which are all soft-ultraluminous sources. In particular, NGC 5907 ULX-1 and NGC 55 ULX-1 are characterized by very soft spectra, where blueshifted $(\sim 0.2 c)$ absorption features are observed and associated with powerful outflows that obscure the inner and hotter regions of the accreting system (e.g., Pinto et al. 2017).

It has been proposed that the short-term variability observed in the soft ULX can be ascribed to the turbulence of the outflows that intersect, from time to time, the LOS. Hence, this leads to the conclusion that the sources are seen from a high inclination angle. We may therefore propose that ULX-1 is inclined so much that our LoS is close to the border of the outflow photosphere, which can randomly obscure/unveil the inner, hotter regions close to the compact object, as in NGC 55 ULX-1. Alternatively, the variability may be ascribed to an unstable mass transfer rate. Furthermore, ULX-1 shows a variability that may resemble some states of the Galactic $\mathrm{BH}$ binary GRS $1915+105$, such as the $\Theta$ or $\rho$ classes (e.g., Belloni et al. 2000), which happen on shorter timescales (the data quality is of course much higher than that of ULX-1). GRS $1915+105$ is a well known swinging Eddington/superEddington accreting source (e.g., Vilhu 1999) and its complex variability was also ascribed to a variable wind (e.g., Neilsen et al. 2012).

Furthermore, the high short-term variability of ULX-1 is similar to that observed in M51 ULX-7 (Liu et al. 2002; Earnshaw et al. 2016; Rodríguez Castillo et al. 2019), which is a PULX and has variability of $30 \%-40 \%$ on timescales of a few kiloseconds as well. This might be an additional hint that ULX-1 hosts an NS in which pulsations are undetected because of the high-inclination viewing angle. Nevertheless, it is important to remark that M51 ULX-7 is spectrally hard, and hence different from ULX-1. However, should the ULX-1 compact object be an NS with a spin period of $\sim 1$ s (as seen in most PULXs), the observed source flux variability could not be ascribed to propeller effects, as it would produce a bimodal luminosity variation of more than two orders of magnitude: $\Delta L \sim 170 P^{2 / 3} M_{1.4 M_{\odot}}^{1 / 3} R_{10^{6} \mathrm{~cm}}^{-1} \sim 170$ (e.g., Corbet 1996; Campana et al. 2001; Mushtukov et al. 2015; Tsygankov et al. 2016; Campana et al. 2018). On the other hand, a period smaller than $1 \mathrm{~s}$ can produce the observed luminosity jump.

Finally, Earnshaw et al. (2019) showed that among a population of $\sim 300$ ULXs, only 8 sources were found to be highly variable (rms $>30 \%$ ) on timescales of hundreds to a few thousands of kiloseconds. Using the XMM-DR4 catalog, we found that if we limit our investigation to the ULXs with observations longer than $30 \mathrm{ks}$, we have 5-6 variable objects, for a total of 182 selected sources. Furthermore, if we consider only the sources with count rates higher than $0.1 \mathrm{cts} \mathrm{s}^{-1}$, the number of variable ULXs is 4 from a total of 23 selected sources. This implies that casual observing might lead to some bias in the detection of periods of high amplitude variability.

\section{2. $U L X-2$}

The source was detected in both XMM-Newton observations at a luminosity of $8-9 \times 10^{39} \mathrm{erg} \mathrm{s}^{-1}$, with a low short-term flux variability (apart for a slight decay in the 2018 data).

Our spectral analysis showed that a phenomenological model given by two thermal components describes its spectrum. Unfortunately, the quality of the 2005 data did not allow us to constrain any spectral variability. From the higher-quality 2018 data, we estimated temperatures of $0.6 \mathrm{keV}$ and $1.3 \mathrm{keV}$ associated with emitting radii of $400-1400 \mathrm{~km}$ and $\sim 100 \mathrm{~km}$, for the DISKBB and BBODYRAD model, respectively. However, we cannot exclude a super-Eddington scenario in which accretion is driven by an advection-dominated disk with a temperature of $\sim 3 \mathrm{keV}$, as observed in other ULXs (e.g., IC 342 X-1, NGC 5643 ULX-1; Gladstone et al. 2009; Pintore et al. 2016).

Because of its hard spectral shape, the source may be classified as a hard-ultraluminous or a broadened disk ULX (Sutton et al. 2013). In a super-Eddington scenario, the source is likely observed from a low-inclination angle, where the LOS enters into the funnel of the outflows (e.g., Middleton et al. 2015a). The nondetection of pulsations does not allow us to establish if the compact object is an NS or a BH. Simultaneous broadband observations with XMM-Newton and NuSTAR will permit us to better constrain the source nature by, for example, testing the existence of a third, high-energy spectral component, usually observed in the PULX spectra and likely arising from column accretion (e.g., Walton et al. 2018) on top of the NSs.

\subsection{Variable ULXS}

In Walton et al. (2011), ULX-3 and ULX-4 were only marginally classified as ULXs since their luminosity was $\sim 10^{39} \mathrm{erg} \mathrm{s}^{-1}$. Nevertheless, the two sources are not detected in our new observation down to a limit of $<2 \times 10^{38} \mathrm{erg} \mathrm{s}^{-1}$, making them candidate transient ULXs (tULXs). By now, only a dozen ULXs are known to be transient; however, casual observational scheduling might have some bias in the observed variability pattern and the detection of periods of high variability in ULXs. Furthermore, most of the tULXs have 
been discovered only by chance (e.g., Soria et al. 2012; Esposito et al. 2013; Middleton et al. 2013; Carpano et al. 2018; Earnshaw et al. 2018; Pintore et al. 2018; van Haaften et al. 2019). Therefore, the actual fraction of tULXs and their duty cycle are poorly constrained. Regular high-quality X-ray monitoring of a large sample of galaxies hosting ULXs (which seemingly are a suitable environment for the production of ULXs) are needed.

We stress that all confirmed PULXs belong to the transient group, and the switch-off may be caused by propeller effects (e.g., Tsygankov et al. 2016; Israel et al. 2017), which cause a drop in luminosity of $\Delta L \sim 170 P^{2 / 3} M_{1.4 M_{\odot}}^{1 / 3} R_{10^{6} \mathrm{~cm}}^{-1}$. Assuming a spin period $\leqslant 1 \mathrm{~s}$, the $3 \sigma$ flux upper limits are compatible with the entrance in the propeller for both ULX-3 and ULX-4. Therefore, it is possible that, even though the pulsations are not detected (perhaps due to limited statistics), ULX-3 and ULX-4 could potentially host NSs. We cannot rule out an alternative explanation for the high level of variability among observation being due to high amplitude super-orbital modulations. Furthermore, another possibility is that these ULXs host BHs and they were observed during a particularly bright outburst (e.g., Esposito et al. 2013; Middleton et al. 2013; Earnshaw et al. 2018)

Finally, similar considerations also apply to the new source (ULX-5), should it be confirmed as a genuine ULX in the NGC 7456 galaxy.

\section{Conclusions}

In this work we have presented a full study of the ULX population in the galaxy NGC 7456. Two of these are bright sources with luminosities of $5 \times 10^{39}-10^{40} \mathrm{erg} \mathrm{s}^{-1}$ (which might be persistent sources) and are spectrally described by a two-component thermal model, as usually seen in ULX spectra. However, at least one of the two (ULX-1) is highly variable (varying by an order of magnitude) on timescales of hundreds of seconds to kiloseconds, presenting one of the largest flux variations ever observed in a ULX $(\sim 50 \%$ fractional variability). Such variability is mainly driven by the highenergy part of the emission. The nature of its compact object is not yet clear. We propose that the source is seen with an inclination angle such that our LOS occasionally straddles the optically thick turbulence of an outflow, which occasionally covers the inner regions where the high-energy emission is produced. Alternatively, the variability may be related to changes in the accretion rate. ULX-1 increases the sample of highly variable sources and we cannot exclude that the compact object is an NS.

ULX-2 is instead spectrally rather hard and can also be modeled by a single thick disk, suggesting a super-Eddington accreting scenario.

Two other ULXs (ULX-3 and ULX-4) in the galaxy are variable, and seem to reach and marginally overcome the ULX threshold. These can be considered transient ULX candidates. Finally, we found a possible new bright source (ULX-5) in the galaxy, the nature of which is not yet constrained. Further observations will be necessary to determine the possible transient nature of these sources.

The scientific results reported in this article are based on observations obtained with XMM-Newton, an ESA science mission with instruments and contributions directly funded by ESA Member States and NASA. L.S. acknowledges support from the research grant "iPeska" (P.I. Andrea Possenti) funded under the INAF national call Prin-SKA/CTA approved with the Presidential Decree 70/2016. G.R. acknowledges the support of high performance computing resources awarded by CINECA (MARCONI and GALILEO), under the ISCRA initiative and the INAF-CIENCA MoU; and also the computing centers of INAF-Osservatorio Astronomico di Trieste and Osservatorio Astrofisico di Catania, under the coordination of the CHIPP project, for the availability of computing resources and support. C.P. is supported by ESA Research Fellowships. D.J.W. and M.M. acknowledges support from STFC in the form of an Ernest Rutherford Fellowship. H.P.E. acknowledges support under NASA contract NNG08FD60C. F.B. is funded by the European Union's Horizon 2020 research and innovation programme under the Marie Skłodowska Curie grant agreement No. 664931. This research was supported by high performance computing resources at New York University Abu Dhabi. H.P.E. acknowledges support under NASA contract NNG08FD60C. T.P.R. acknowledges support from STFC as part of the consolidated grant ST/K000861/1. A.P. acknowledges financial support from the Italian Space Agency and National Institute for Astrophysics, ASI/INAF, under agreements ASI-INAF I/037/12/0 and ASI-INAF n.2017-14-H.0.

We acknowledge funding in the framework of the project ULTraS ASI-INAF contract No. 2017-14-H.0, and project ASI-INAF contract I/037/12/0.

\section{ORCID iDs}

M. Marelli (ib https://orcid.org/0000-0002-8017-0338

R. Salvaterra (i) https://orcid.org/0000-0002-9393-8078

G. L. Israel (i) https://orcid.org/0000-0001-5480-6438

P. Esposito (1D https://orcid.org/0000-0003-4849-5092

A. Belfiore (ib https://orcid.org/0000-0002-2526-1309

S. Mereghetti (iD https://orcid.org/0000-0003-3259-7801

L. Stella (10) https://orcid.org/0000-0002-0018-1687

H. P. Earnshaw (1) https://orcid.org/0000-0001-5857-5622

T. P. Roberts (i) https://orcid.org/0000-0001-8252-6337

D. J. Walton (iD https://orcid.org/0000-0001-5819-3552

F. Bernardini (iD https://orcid.org/0000-0001-5326-2010

F. Haberl (i) https://orcid.org/0000-0002-0107-5237

A. Tiengo (ii) https://orcid.org/0000-0002-6038-1090

L. Zampieri it https://orcid.org/0000-0002-6516-1329

M. Bachetti (i) https://orcid.org/0000-0002-4576-9337

D. D’Agostino (iD https://orcid.org/0000-0003-2649-0071

F. Fürst (iD https://orcid.org/0000-0003-0388-0560

M. Mapelli (iD https://orcid.org/0000-0001-8799-2548

A. Papitto (iD https://orcid.org/0000-0001-6289-7413

M. Middleton (i) https://orcid.org/0000-0002-8183-2970

\section{References}

Abbott, B. P., Abbott, R., Abbott, T. D., et al. 2016, PhRvL, 116, 061102 Abbott, B. P., Abbott, R., Abbott, T. D., et al. 2019, ApJ, 882, L24

Arnaud, K. A. 1996, in ASP Conf. Ser. 101, Astronomical Data Analysis Software and Systems V, ed. G. H. Jacoby \& J. Barnes (San Francisco, CA: ASP), 17

Bachetti, M., Rana, V., Walton, D. J., et al. 2013, ApJ, 778, 163 Barnard, R. 2010, MNRAS, 404, 42

Belfiore, A., Esposito, P., Pintore, F., et al. 2020, NatAs, 4, 147

Belloni, T., Klein-Wolt, M., Méndez, M., van der Klis, M., \& van Paradijs, J. 2000, A\&A, 355, 271

Belloni, T. M., Motta, S. E., \& Muñoz-Darias, T. 2011, BASI, 39, 409

Brightman, M., Harrison, F. A., Fürst, F., et al. 2018, NatAs, 2, 312

Campana, S., Gastaldello, F., Stella, L., et al. 2001, ApJ, 561, 924 
Campana, S., Stella, L., Mereghetti, S., \& de Martino, D. 2018, A\&A, 610, A46

Carpano, S., Haberl, F., Maitra, C., \& Vasilopoulos, G. 2018, MNRAS, 476, 45

Colbert, E. J. M., \& Mushotzky, R. F. 1999, ApJ, 519, 89

Corbet, R. H. D. 1996, ApJ, 457, L31

Coti Zelati, F., Rea, N., Pons, J. A., Campana, S., \& Esposito, P. 2018, MNRAS, 474, 961

De Luca, A., Tiengo, A., D'Agostino, D., et al. 2016, in XMM-Newton: The Next Decade (Paris: ESA), 42

Earnshaw, H. M., Roberts, T. P., Heil, L. M., et al. 2016, MNRAS, 456, 3840

Earnshaw, H. P., Grefenstette, B. W., Brightman, M., et al. 2019, ApJ, 881, 38

Earnshaw, H. P., Roberts, T. P., Middleton, M. J., Walton, D. J., \& Mateos, S. 2019, MNRAS, 483, 5554

Earnshaw, H. P., Roberts, T. P., \& Sathyaprakash, R. 2018, MNRAS, 476, 4272

Esposito, P., Motta, S. E., Pintore, F., Zampieri, L., \& Tomasella, L. 2013, MNRAS, 428, 2480

Fabbiano, G. 1989, ARA\&A, 27, 87

Farrell, S. A., Webb, N. A., Barret, D., Godet, O., \& Rodrigues, J. M. 2009, Nature, 460, 73

Feng, H., \& Soria, R. 2011, NewAR, 55, 166

Fürst, F., Walton, D. J., Harrison, F. A., et al. 2016, ApJ, 831, L14

Gladstone, J. C., Roberts, T. P., \& Done, C. 2009, MNRAS, 397, 1836

Heil, L. M., Vaughan, S., \& Roberts, T. P. 2009, MNRAS, 397, 1061

HI4PI Collaboration 2016, A\&A, 594, A116

Israel, G. L., Belfiore, A., Stella, L., et al. 2017, Sci, 355, 817

Israel, G. L., Papitto, A., Esposito, P., et al. 2017, MNRAS, 466, L48

Israel, G. L., \& Stella, L. 1996, ApJ, 468, 369

Kaaret, P., Feng, H., \& Roberts, T. P. 2017, ARA\&A, 55, 303

Kawaguchi, T. 2003, ApJ, 593, 69

King, A. R. 2009, MNRAS, 393, L41

Koliopanos, F., Vasilopoulos, G., Godet, O., et al. 2017, A\&A, 608, A47

Kosec, P., Pinto, C., Walton, D. J., et al. 2018, MNRAS, 479, 3978

Liu, J.-F., Bregman, J. N., \& Seitzer, P. 2002, ApJ, 580, L31

Maccacaro, T., Gioia, I. M., Wolter, A., Zamorani, G., \& Stocke, J. T. 1988, ApJ, 326, 680

Madsen, K. K., Harrison, F. A., Markwardt, C. B., et al. 2015, ApJS, 220, 8

Mapelli, M., Annibali, F., Zampieri, L., \& Soria, R. 2013, MNRAS, 433, 849

Mapelli, M., Colpi, M., \& Zampieri, L. 2009, MNRAS, 395, L71

Mapelli, M., Ripamonti, E., Zampieri, L., Colpi, M., \& Bressan, A. 2010, MNRAS, 408, 234

Marelli, M., Tiengo, A., De Luca, A., et al. 2017, ApJ, 851, L27

McClintock, J. E., \& Remillard, R. A. 2006, in Compact Stellar X-ray Sources, ed. W. H. G. Levin \& M. van der Klis (Cambridge: Cambridge Univ. Press), 157

McMahon, R. G., Banerji, M., Gonzalez, E., et al. 2013, Msngr, 154, 35

Middleton, M. J., Brightman, M., Pintore, F., et al. 2019, MNRAS, 486, 2

Middleton, M. J., Heil, L., Pintore, F., Walton, D. J., \& Roberts, T. P. 2015a, MNRAS, 447, 3243

Middleton, M. J., \& King, A. 2017, MNRAS, 470, L69
Middleton, M. J., Miller-Jones, J. C. A., Markoff, S., et al. 2013, Nature, 493, 187

Middleton, M. J., Walton, D. J., Fabian, A., et al. 2015b, MNRAS, 454, 3134 Mitsuda, K., Inoue, H., Koyama, K., et al. 1984, PASJ, 36, 741

Moretti, A., Campana, S., Lazzati, D., \& Tagliaferri, G. 2003, ApJ, 588, 696 Mushtukov, A. A., Suleimanov, V. F., Tsygankov, S. S., \& Poutanen, J. 2015, MNRAS, 454, 2539

Neilsen, J., Petschek, A. J., \& Lee, J. C. 2012, MNRAS, 421, 502

Olausen, S. A., \& Kaspi, V. M. 2014, ApJS, 212, 6

Pakull, M. W., \& Mirioni, L. 2002, arXiv:astro-ph/0202488

Paturel, G., Petit, C., Prugniel, P., et al. 2003, A\&A, 412, 45

Pinto, C., Alston, W., Soria, R., et al. 2017, MNRAS, 468, 2865

Pinto, C., Middleton, M. J., \& Fabian, A. C. 2016, Nature, 533, 64

Pintore, F., Belfiore, A., Novara, G., et al. 2018, MNRAS, 477, L90

Pintore, F., Di Salvo, T., Bozzo, E., et al. 2015, MNRAS, 450, 2016

Pintore, F., Zampieri, L., Stella, L., et al. 2017, ApJ, 836, 113

Pintore, F., Zampieri, L., Sutton, A. D., et al. 2016, MNRAS, 459, 455

Plotkin, R. M., Gallo, E., \& Jonker, P. G. 2013, ApJ, 773, 59

Poutanen, J., Lipunova, G., Fabrika, S., Butkevich, A. G., \& Abolmasov, P. 2007, MNRAS, 377, 1187

Pye, J. P., Rosen, S., Fyfe, D., \& Schröder, A. C. 2015, A\&A, 581, A28

Rea, N., \& Esposito, P. 2011, in High-Energy Emission from Pulsars and Their Systems, ed. D. F. Torres \& N. Rea (Berlin: Springer), 247

Rigoselli, M., \& Mereghetti, S. 2018, A\&A, 615, A73

Rodríguez Castillo, G. A., Israel, G. L., Belfiore, A., et al. 2019, A\&A, submitted (arXiv:astro-ph.HE/1906.04791)

Sathyaprakash, R., Roberts, T. P., Walton, D. J., et al. 2019, MNRAS, 488, 35

Song, X., Walton, D. J., Lansbury, G. B., et al. 2020, MNRAS, 491, 1260

Soria, R., Kuntz, K. D., Winkler, P. F., et al. 2012, ApJ, 750, 152

Stobbart, A.-M., Roberts, T. P., \& Warwick, R. S. 2004, MNRAS, 351, 1063

Stobbart, A.-M., Roberts, T. P., \& Wilms, J. 2006, MNRAS, 368, 397

Sutton, A. D., Roberts, T. P., \& Middleton, M. J. 2013, MNRAS, 435, 1758

Takeuchi, S., Ohsuga, K., \& Mineshige, S. 2013, PASJ, 65, 88

Tsygankov, S. S., Mushtukov, A. A., Suleimanov, V. F., \& Poutanen, J. 2016, MNRAS, 457, 1101

Tully, R. B., Courtois, H. M., \& Sorce, J. G. 2016, AJ, 152, 50

van Haaften, L. M., Maccarone, T. J., Rhode, K. L., Kundu, A., \& Zepf, S. E. 2019, MNRAS, 483, 3566

Vaughan, S., Edelson, R., Warwick, R. S., \& Uttley, P. 2003, MNRAS, 345,1271

Vilhu, O. 1999, in ASP Conf. Ser. 161, High Energy Processes in Accreting Black Holes, Super-Eddington Accretion in GRS 1915+105, ed. J. Poutanen \& R. Svensson (San Francisco, CA: ASP), 82

Walton, D. J., Fürst, F., Harrison, F. A., et al. 2018, MNRAS, 473, 4360

Walton, D. J., Roberts, T. P., Mateos, S., \& Heard, V. 2011, MNRAS, 416, 1844

Wiktorowicz, G., Sobolewska, M., Lasota, J.-P., \& Belczynski, K. 2017, ApJ, 846,17

Wilson-Hodge, C. A., Malacaria, C., Jenke, P. A., et al. 2018, ApJ, 863, 9

Zampieri, L., \& Roberts, T. P. 2009, MNRAS, 400, 677 